\title{
Dynamics of Hollow Atom Formation in Intense X-Ray Pulses Probed by Partial Covariance Mapping
}

L. J. Frasinski, ${ }^{1, *}$ V. Zhaunerchyk,${ }^{2}$ M. Mucke, ${ }^{2}$ R. J. Squibb, ${ }^{1,2}$ M. Siano, ${ }^{1}$ J. H. D. Eland, ${ }^{2,3}$ P. Linusson, ${ }^{4}$ P. v.d. Meulen, ${ }^{4}$ P. Salén, ${ }^{4}$ R. D. Thomas, ${ }^{4}$ M. Larsson, ${ }^{4}$ L. Foucar, ${ }^{5,6}$ J. Ullrich, ${ }^{5,7,8}$ K. Motomura, ${ }^{9}$ S. Mondal, ${ }^{9}$ K. Ueda, ${ }^{9}$ T. Osipov, ${ }^{10}$ L. Fang, ${ }^{10}$ B. F. Murphy, ${ }^{10}$ N. Berrah, ${ }^{10}$ C. Bostedt, ${ }^{11}$ J. D. Bozek, ${ }^{11}$ S. Schorb, ${ }^{11}$ M. Messerschmidt, ${ }^{11}$ J. M. Glownia, ${ }^{11}$ J. P. Cryan, ${ }^{11}$ R. N. Coffee, ${ }^{11}$ O. Takahashi, ${ }^{12}$ S. Wada, ${ }^{13}$ M. N. Piancastelli, ${ }^{2,14}$ R. Richter,${ }^{15}$ K. C. Prince, ${ }^{15}$ and R. Feifel ${ }^{2, \dagger}$

${ }^{1}$ Blackett Laboratory, Imperial College London, London SW7 2AZ, United Kingdom

${ }^{2}$ Department of Physics and Astronomy, Uppsala University, SE-751 20 Uppsala, Sweden

${ }^{3}$ Physical and Theoretical Chemistry Laboratory, Oxford University, Oxford OX1 3QZ, United Kingdom

${ }^{4}$ Department of Physics, Stockholm University, SE-106 91 Stockholm, Sweden

${ }^{5}$ Advanced Study Group of the Max Planck Society, 22607 Hamburg, Germany

${ }^{6}$ Max Planck Institut für medizinische Forschung, 69120 Heidelberg, Germany

${ }^{7}$ Max Planck-Institut für Kernphysik, 69117 Heidelberg, Germany

${ }^{8}$ Physikalisch-Technische Bundesanstalt, 38116 Braunschweig, Germany

${ }^{9}$ Institute for Interdisciplinary Material Research, Tohoku University, Sendai 980-8577, Japan

${ }^{10}$ Department of Physics, Western Michigan University, Kalamazoo, Michigan 49008, USA

${ }^{11}$ SLAC National Accelerator Laboratory, Menlo Park, California 94025, USA

${ }^{12}$ Department of Chemistry, Hiroshima University, Higashi-Hiroshima 739-8526, Japan

${ }^{13}$ Department of Physical Science, Hiroshima University, Higashi-Hiroshima 739-8526, Japan

${ }^{14}$ Laboratoire de Chimie Physique-Matière et Rayonnement, Université Pierre et Marie Curie, 75231 Paris, France

${ }^{15}$ Elettra-Sincrotrone Trieste, 34149 Trieste, Italy

(Received 10 April 2013; published 13 August 2013)

\begin{abstract}
When exposed to ultraintense $\mathrm{x}$-radiation sources such as free electron lasers (FELs) the innermost electronic shell can efficiently be emptied, creating a transient hollow atom or molecule. Understanding the femtosecond dynamics of such systems is fundamental to achieving atomic resolution in flash diffraction imaging of noncrystallized complex biological samples. We demonstrate the capacity of a correlation method called "partial covariance mapping" to probe the electron dynamics of neon atoms exposed to intense $8 \mathrm{fs}$ pulses of $1062 \mathrm{eV}$ photons. A complete picture of ionization processes competing in hollow atom formation and decay is visualized with unprecedented ease and the map reveals hitherto unobserved nonlinear sequences of photoionization and Auger events. The technique is particularly well suited to the high counting rate inherent in FEL experiments.
\end{abstract}

Imaging at the atomic level can use very short, intense $\mathrm{x}$-ray pulses from a free electron laser (FEL) [1] to record the diffraction pattern from biological molecules before they explode due to massive photoionization [2]. Such imaging has been demonstrated with resolution of tens of nanometers [3], but atomic resolution will require understanding the dynamics of fast multiphoton ionization from inner shells. This can create hollow atoms [4,5] and highly charged species [6] which modify the diffraction pattern [7]. In this Letter we demonstrate the capacity of covariance mapping [8] to reveal the electron dynamics of multiphoton hollow atom formation at FELs [9]. We introduce partial covariance so compensating for the strong fluctuations in pulse energy, which spoil conventional covariance maps. The technique allows us to identify hitherto unobserved photoionization and Auger sequences, their branching ratios and femtosecond dynamics.

While some useful information on electron dynamics in intense x-ray fields can be obtained by monitoring the ion charge states [5], this information is incomplete because different ionization sequences can lead to the same product. In particular, for the formation of transient hollow atoms which cannot be discerned this way, electron energy spectra are required. High charge states produced in such experiments generate spectra where different electron energies overlap and can be untangled only in special cases [5]. An unambiguous identification of ionization sequences requires correlation of at least pairs of electron energies. Coincidence techniques [10] can reveal such correlations but require low counting rates, typically 1 coincidence event per 10 to 100 radiation pulses. Reducing the data acquisition to such a low level [11] is impracticable at FELs, which are single-user facilities operating at low repetition rates. To overcome these experimental obstacles we present an enhanced version of the covariance mapping technique [8].

The experiment was performed using the Atomic, Molecular, and Optical science instrument of the Linac Coherent Light Source (LCLS) FEL at the SLAC National Accelerator Laboratory, Stanford [12]. X-rays are focused on a pulsed jet of $\mathrm{Ne}$ atoms and the emitted electrons are 
guided by the field lines of a long magnetic bottle spectrometer [13], custom-made for the present purpose, towards the detector. A transient digitizer records a timeof-flight (TOF) spectrum of electrons created at each laser shot and sends it to fast data storage and to a data analysis system [14], which converts the spectra from TOF to energy and calculates the covariance map. Both the timeaveraged electron energy spectrum and the corresponding covariance map are displayed in real time to monitor the data quality. (More experimental details including a schematic figure, Fig. S1, are given in the Supplemental Material [15]).

If two or more electrons are liberated from the target by the X-ray pulse and one characteristic electron is detected at energy $E_{x}$ there is a higher than average probability of detecting a second electron from the same event at another energy $E_{y}$. The existence of the correlated electrons can be revealed by calculating the covariance of the signal at $E_{x}$ with the signal at other energies, which include $E_{y}$. Formally this means taking a row vector of the single-shot energy spectrum, $\mathbf{X}\left(E_{x}\right)$, transposing it into a column copy, $\mathbf{Y}\left(E_{y}\right)=\mathbf{X}\left(E_{x}\right)^{T}$, and calculating a covariance matrix

$$
\operatorname{cov}(\mathbf{Y}, \mathbf{X})=\langle\mathbf{Y} \mathbf{X}\rangle-\langle\mathbf{Y}\rangle\langle\mathbf{X}\rangle
$$

where \langle\rangle denotes an average taken over many laser shots (see Fig. S2 of the Supplemental Material [15] for instances of the single-shot spectra; note that the counting rate is typically 30 electrons per radiation pulse-300 to 3000 -fold higher than in coincidence techniques). The covariance matrix can be visualized as a map, as shown in Fig. 1, of (at least) twoelectron processes, which appear as positive features. The important property of the covariance estimator (unlike a correlation coefficient) is that the feature volumes are directly proportional to the probability of the underlying physics. This property originates in Poissonian fluctuations of the number of atoms in the focal region and is related to the fact that the variance of a Poissonian process is equal to its mean.

The simple covariance technique outlined above often produces artificial correlations which are not of physical interest. For example, if the laser pulse energy increases from one shot to the next, there are more electrons produced in every process, and every feature on the map becomes correlated with every other via the common influence of laser fluctuations. Usually, it is impossible to keep the experimental conditions exactly the same from shot to shot and the only way to suppress the unwanted common correlations is to reduce the counting rate and run the experiment for a longer time. In practice, the FEL X-ray pulses fluctuate widely (see Fig. S3 of the Supplemental Material [15]).

To accommodate the high counting rate characteristic for FEL experiments we enhance the simple covariance mapping technique by monitoring the X-ray pulse energy, $I$, at every shot and use this information to calculate partial covariance [16]

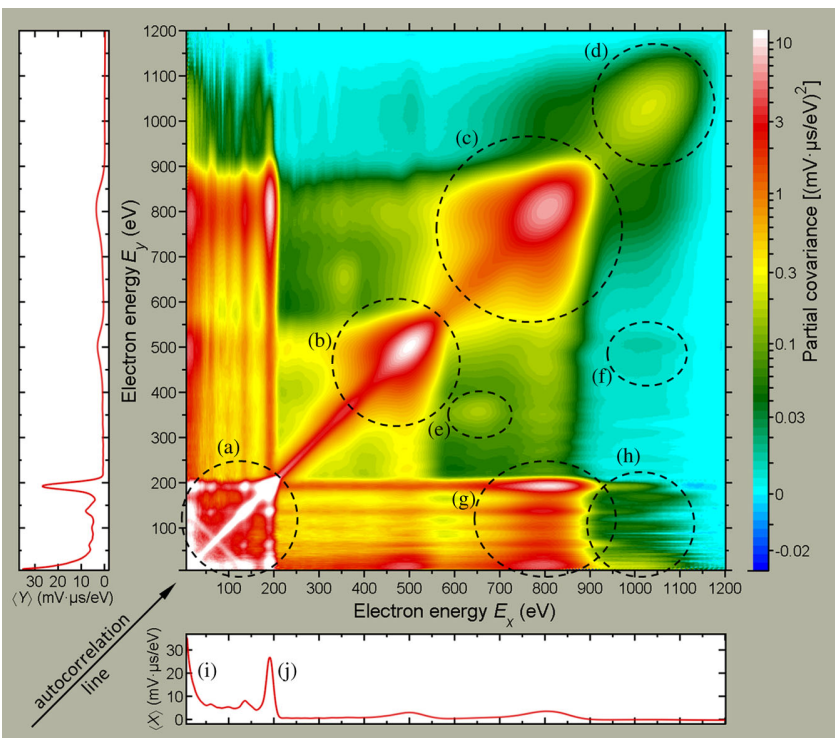

FIG. 1 (color online). A partial covariance map revealing correlations between electrons emitted from neon (and from some $\mathrm{N}_{2}$ and water vapor contamination). The map is constructed shot by shot from electron energy spectra recorded at the photon energy of $1062 \mathrm{eV}$, which are shown along the $x$ and $y$ axes after averaging over 480000 FEL shots. Volumes of the features on the map give relative probabilities of various ionization sequences, which can be classified as: (a) Ne core-core; (b) $\mathrm{H}_{2} \mathrm{O}$ core-core, core-Auger, and Auger-Auger; (c) Ne AugerAuger; (d) Ne valence-valence; (e) $\mathrm{N}_{2}$ core-Auger; (f) $\mathrm{H}_{2} \mathrm{O}$ corevalence; (g) Ne core-Auger; (h) Ne core-valence; (i) double Auger and secondary electrons from electrode surfaces; and (j) Ne main (core) photoelectron line. Note that the gray scale (false-color scale) is nonlinear to accommodate a large dynamic range.

$\operatorname{pcov}(\mathbf{Y}, \mathbf{X} ; I)=\operatorname{cov}(\mathbf{Y}, \mathbf{X})-\operatorname{cov}(\mathbf{Y}, I) \operatorname{cov}(I, \mathbf{X}) / \operatorname{cov}(I, I)$

The second term in this formula can be regarded as a correction for fluctuations in $I$. The result is equivalent to holding the pulse energy constant, inducing only a little extra noise due to the statistical origin of the correction. Equation (2) can be extended easily to compensate for more than one influence on the raw signals, provided the relevant parameters are monitored at every shot.

Equation (2) compensates for unwanted correlations linear with $I$. It also compensates for the majority of nonlinear correlations induced by $I$ because the signal is corrected at its mean level rather than at zero. In fact, any uncompensated nonlinear artifacts are not discernible under our experimental conditions, confirming that Eq. (2) is applicable to quite large fluctuations of the pulse energy (see Fig. S3 of the Supplemental Material [15]).

We have chosen neon as a showcase system because it is the simplest atom where a plethora of single-photon and multiphoton sequential absorption and decay processes are possible. In Fig. 2 we show schematics of multiphoton 


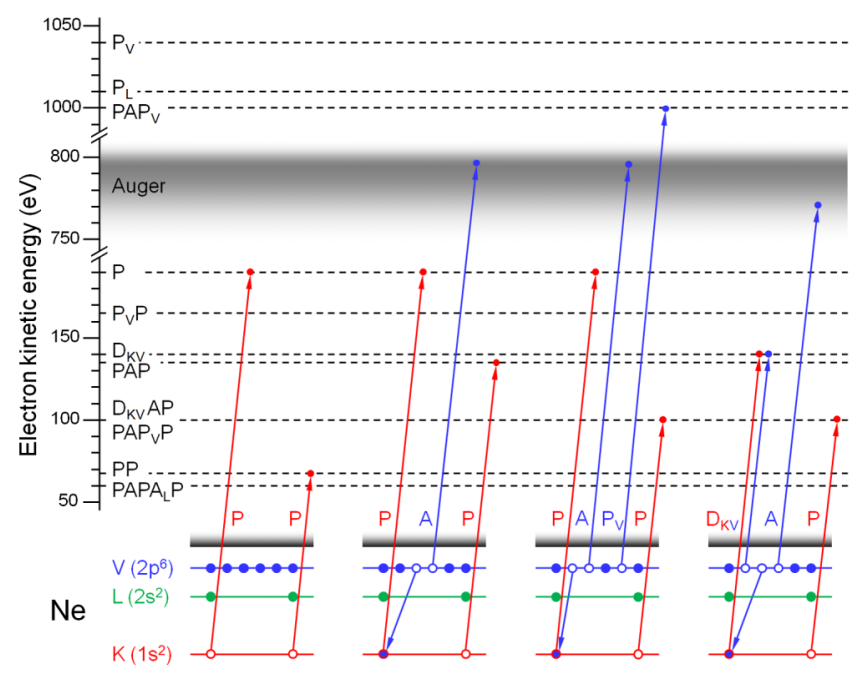

FIG. 2 (color online). Ionization processes in neon induced by intense $1062 \mathrm{eV}$ photons. The processes are ordered according to the kinetic energy of the final electron. Four examples of these processes are shown diagrammatically below. The first process (PP) competes with the second one (PAP). Covariance mapping is required to separate the last two processes $\left(\mathrm{PAP}_{V} \mathrm{P}\right.$ and $\left.\mathrm{D}_{K V} \mathrm{AP}\right)$ because the final kinetic energy is the same.

processes and related kinetic energies of the emitted photoand/or Auger electrons. We demonstrate that the signatures for all of the sequences illustrated are present in our covariance maps, which provide the unique capability to distinguish them in an efficient way.

Figure 1 shows an overview partial covariance map of neon recorded at the photon energy of $1062 \mathrm{eV}$ (with a nominal pulse duration of $8 \mathrm{fs}$ and a nominal pulse energy of $0.11 \mathrm{~mJ}$ ) which was chosen to access the core $(1 \mathrm{~s})$ electrons of the neutral species $(870.2 \mathrm{eV}$ binding energy [17]). The off-diagonal features are associated with pairwise correlations of the electrons ejected upon sequential $\mathrm{x}$-ray multiphoton absorption by Ne atoms. Conventional, one-dimensional electron energy spectra are shown along the $x$ and $y$ axes. The peaks discernible in these spectra are revealed as subdivided into several more features on the map. For example, the 0-200 eV kinetic energy part reflects features associated with core-core, core-Auger, and core-valence processes according to their inherent electron-electron correlations.

The map is symmetric about the diagonal because the same spectra are used for both axes. The strong autocorrelation line on the diagonal has the same origin: an electron detected with energy $E_{x}$ will always be present in the covariance map at $E_{y}=E_{x}$, unlike electrons with different energies, which have the detection and collection efficiency factored in. The symmetry and the autocorrelation line are not present if different $x, y$ spectra are used, for example, in electron-ion covariance mapping [18].

Ionization of background gases, such as water or nitrogen, is displaced from neon processes in the map and does not interfere with the analysis. The $\mathrm{N}_{2}$ core-Auger island was useful for calibration of the TOF-to-energy conversion (see the Supplemental Material [15]).

The x-ray pulses suffer from photon energy jitter, typically $0.5 \%$ of the nominal photon energy. The actual photon energy can be derived from the FEL electron beam energy, which is measured for every shot. This makes it possible to compensate single-shot spectra for the jitter before using them to construct the map. The compensation sharpens photoelectron peaks but broadens Auger ones (if they cannot be treated separately). On the map it removes all distortions in the most interesting core-core region, but leaves some residual distortion in the core-Auger region.

The Ne core-core and core-valence regions are magnified in Fig. 3. The autocorrelation line dominates the map near the diagonal and secondary electrons released from the surface of the permanent magnet near the focal spot mask other features below $30 \mathrm{eV}$. A summary of the identified pairwise electron correlations, with the kinetic energies and assignments, is given in Table I.

Among the dynamical processes depicted in Fig. 3, a likely ionization sequence is the photoelectron-Auger electron-photoelectron-Auger electron (PAPA) process [21], where a photoelectron $(\mathrm{P})$ is ejected from the core, followed by ejection of an Auger electron (A), followed by PA again. This process gives rise to the relatively intense peaks at the energy positions $(136 \mathrm{eV}, 192 \mathrm{eV})$ labeled in Figs. 2 and 3 as PAP. Here, and henceforward, we use the notation $1 s=K, 2 s=L$, and $2 p=V$ to label the orbitals. When $\mathrm{P}$ is without a subscript it means ejection from the $K$ shell. Similarly, when $A$ is without a subscript, it refers to an Auger process involving two electrons from the $V$ shell. The two detected particles giving rise to the covariance signal are indicated by bold type.

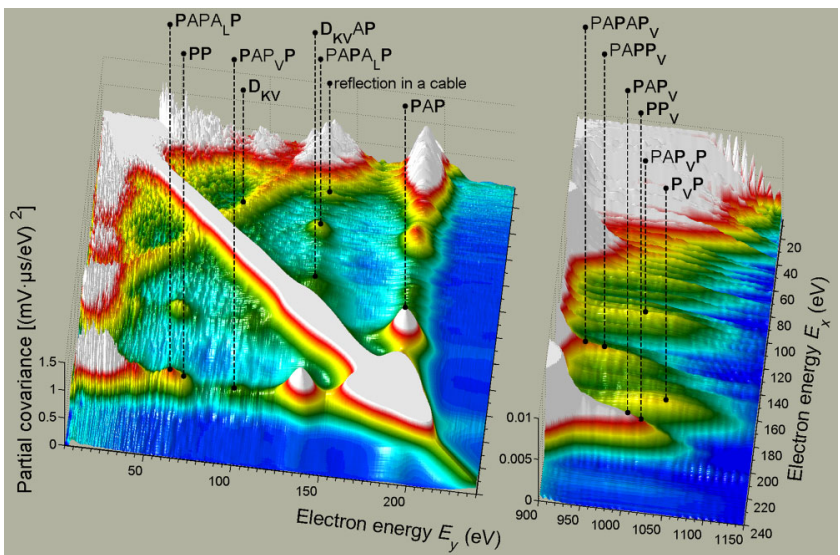

FIG. 3 (color online). Identification of neon ionization processes in the core-core (left) and core-valence (right) correlation regions. The top of the autocorrelation line is cutoff to show the features behind. Symbols P, D, and A denote, respectively, ejection of a photoelectron, two photoelectrons (direct double photoionization by a single photon), and an Auger electron. The other notation used is explained in the text. 
TABLE I. Coordinates of correlation islands revealed in Figs. 1 and 3. Bold type indicates the correlated electron pair. Our measurements are compared with theoretical [19] and other experimental $[17,20]$ data.

\begin{tabular}{|c|c|c|}
\hline \multirow[b]{2}{*}{ Process } & \multicolumn{2}{|c|}{ Kinetic energy of electron pairs $(\mathrm{eV})$} \\
\hline & This work & Other work \\
\hline $\mathbf{P A P}$ & $(192 \pm 7,136 \pm 6)$ & $(192$ [17], 132 [19]) \\
\hline $\mathbf{P P}$ & $(191 \pm 7,60 \pm 4)$ & $(192[17], 69$ [19]) \\
\hline $\mathbf{P A P}_{V} \mathbf{P}$ & $(193 \pm 7,95 \pm 7)$ & $(192$ [17], 103 [19]) \\
\hline $\mathrm{PAPA}_{L} \mathbf{P}$ & $(138 \pm 6,60 \pm 6)$ & (132 [19], 66 [19]) \\
\hline $\mathbf{D}_{K V}$ & Sum $=143 \pm 5$ & Sum $=142[20]$ \\
\hline $\mathbf{P}_{V} \mathbf{P}$ & $(1049 \pm 15,169 \pm 5)$ & $(1040$ [17], 167 [19]) \\
\hline $\mathrm{PAP}_{V} \mathbf{P}$ & $(1024 \pm 15,103 \pm 4)$ & $(1000[19], 103$ [19]) \\
\hline $\mathbf{D}_{K V} \mathrm{~A} \mathbf{P}$ & $(138 \pm 5,101 \pm 6)$ & $(142[20], 103$ [19]) \\
\hline
\end{tabular}

At a pulse duration larger than the core hole lifetime, a less likely process is PPAA, where a second photoelectron is ejected before the first hole is filled thereby creating a transient hollow atom which is filled in a subsequent AA process. Its lower probability is reflected in a smaller height of the PP feature in Fig. 3. The ratio of the integrated intensity of this feature to that of the $\mathbf{P A P}$ feature is measured as 0.28 . The shorter the FEL pulse, however, the more probable is the PP process. To observe this effect we have analyzed the covariance map according to the $\mathrm{x}$-ray pulse duration, as estimated from the charge-to-current ratio recorded for each electron bunch (this duration is probably an overestimate because the $\mathrm{x}$-ray pulses have substructures [1]). In Fig. 4(a) we plotted the integrated volumes of two areas in the covariance map [region $(\mathrm{g})$ of Fig. 1] which correspond to a one-photon PA process and a two-photon PPA process, respectively. In both cases the first photoelectron and the associated Auger electron were detected. As can be seen, the probability of hollow atom formation decreases with increasing pulse duration, while the PA process, whose probability depends only on atomic properties, is little affected by the pulse duration (the small observed variation of the PA process with the pulse duration is probably due to a residual correlation between the pulse duration and energy).

After the PAPA process another core ionization is still energetically possible, which we observe in Fig. 3 as the $\mathbf{P A P A}_{\mathrm{L}} \mathbf{P}$ and $\mathrm{PAPA}_{\mathrm{L}} \mathbf{P}$ islands of the same PAPAP sequence but with different photoelectrons detected. An Auger process involving the $L$ shell is a minor channel in $\mathrm{Ne}^{+}$with a $K$ hole $(K V V: K L V: K L L=60: 25: 5$ [20]), but as the number of ionization steps increases, there is an increasing probability that one of the steps leaves a hole in the $L$ shell; indeed, this seems to be the case here, which is labeled by the subscript $L$. However, the $L$ shell vacancy can also be produced in a shakeup process at the middle $P$. From the significant difference in heights of the $\mathbf{P A P A}_{\mathrm{L}} \mathbf{P}$ and $\mathrm{PAPA}_{\mathrm{L}} \mathbf{P}$ islands we infer that the former has some contribution from the $2 p \rightarrow 3 p$ shakeup satellite of the PAP process.
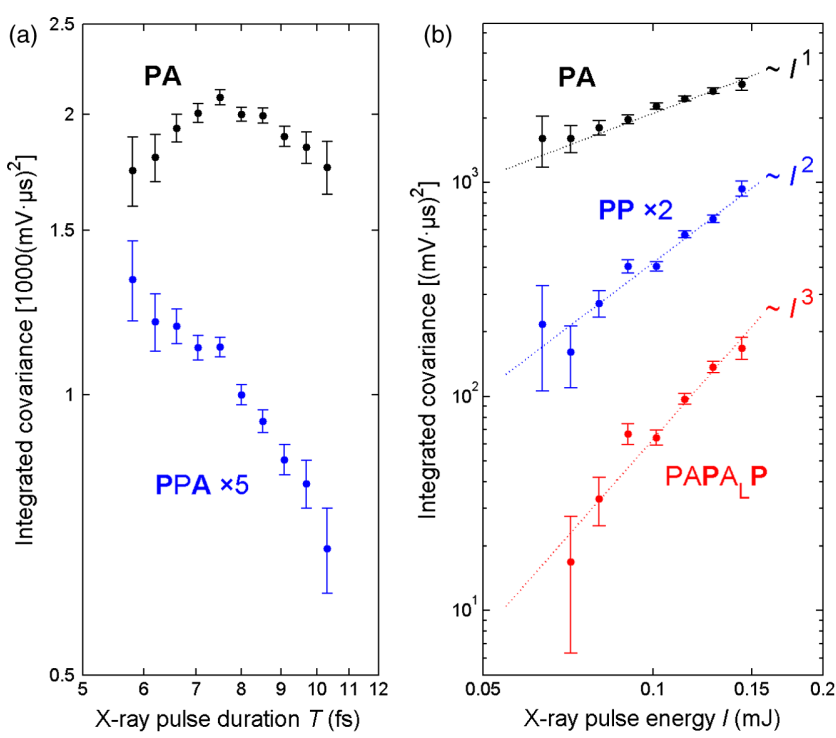

FIG. 4 (color online). Dynamic effects reflected in relative yields of ionization sequences. The yields are integrated volumes of covariance features, such as shown in Figs. 1 and 3. Increasing pulse duration (a) hardly affects the dominant photoelectronAuger (PA) sequence, but reduces the probability of hollow atom formation (PPA) because it has to compete with the former process. For increasing x-ray pulse energy (b) the yields follow a power law whose exponent is the number of photons absorbed in the process. The values of pulse duration and energy are accurate within $\pm 50 \%$. All errors are \pm 1 standard deviation.

The identification and direct comparison of such sequences is a unique asset of our method, while in conventional photoelectron spectra the PAP processes give rise to broad fluctuations over a diffuse background [22].

The ridge labeled as $\mathbf{D}_{\mathrm{KV}}$ in Fig. 3 shows correlation between two photoelectrons simultaneously ejected from the core and valence shells upon absorption of a single photon [23]. The available energy is arbitrarily shared between the two electrons giving a line $E_{x}+E_{y}=$ const, with an increasing height towards the ends of the line due to a tendency towards unequal energy sharing. This higher probability at the ends of the line is also reflected in the nonlinearly continued DAP ionization sequence, which is revealed in this part of the map as a diffuse $\mathbf{D}_{\mathrm{KV}} \mathrm{AP}$ island. This is an example of a hitherto unobserved ionization sequence which can be separated by the present technique from the competing PA $\mathbf{P}_{\mathrm{V}} \mathbf{P}$ sequence, both of which give rise to the same kinetic energy of the final electron.

In addition, several new photoionization processes are revealed in the high energy part of the map in Fig. 3. Notably, covariance mapping unambiguously identifies nonlinear sequences leading to the same final state, for example, the $\mathbf{P}_{V} \mathbf{P}$ island is separate from its chronologically reversed counterpart $\mathbf{P} \mathbf{P}_{\mathbf{V}}$, and $\mathbf{P A} \mathbf{P}_{\mathbf{V}} \mathbf{P}$ is separate from $\mathrm{PAPP}_{\mathbf{V}}$.

The ionization probability is related to the number of photons in a pulse. Specifically, the probability of a process 
involving $n$ photons depends on the $n$th power of the pulse energy. Fig. 4(b) shows examples of such variation (a saturation effect reduces the slope of the PA process somewhat below the theoretical value). Plotting such variations helps considerably to interpret covariance maps.

In conclusion, we have investigated the dynamics of hollow atom formation by resolving on a partialcovariance map different photoionization sequences (such as PAPA and PPAA) leading to the same ion charge state $\left(\mathrm{Ne}^{4+}\right)$. The nature of this technique allowed us to completely quantify the competition between these processes on the femtosecond time scale. On the covariance map we discern several new sequences which give the same kinetic energy of the final electrons and so could not be distinguished previously. We expect that this method of revealing ionization dynamics in intense $x$-ray pulses will help to interpret diffraction patterns and improve the accuracy of structural analysis of complex systems.

The scientific advances we have made validate partial covariance mapping as a practical method of FEL data analysis. It makes it possible to collect much more data within the typical acquisition time available at an FEL, and by its selectivity can cope with the presence of some background impurities. There are good prospects for discerning other multiple core vacancy processes such as two-site double core hole formation $[22,24,25]$ or PAP sequences involving different atomic sites in molecules and using this information for chemical analysis with unprecedented ease.

This work has been financially supported by the Swedish Research Council, the Göran Gustafsson Foundation (UU/ $\mathrm{KTH}$ ), and the Knut and Alice Wallenberg Foundation, Sweden. L. J.F. and R.J.S. thank the EPSRC, UK (Grants No. EP/F021232/1, No. EP/F034601/1, and No. EP/I032517/1). K.C.P. and R. R. acknowledge the MIUR, Italy (Grants No. FIRB-RBAP045JF2 and No. FIRB-RBAP06AWK3). K. U. and K. M. are grateful to MEXT for funding the XFEL Utilization Research Project and the XFEL Priority Strategy Program. S. M. is grateful to JSPS for financial support. N. B., T. O., L. F., and B.F.M. acknowledge financial support by the U.S. Department of Energy, Office of Science, Basic Energy Science, Chemical, Geosciences, and Biological Divisions. Parts of this research were carried out at the Linac Coherent Light Source (LCLS) at the SLAC National Accelerator Laboratory. LCLS is an Office of Science User Facility operated for the U.S. Department of Energy, Office of Science, by Stanford University.

Note added.-In a separate work (which was prepared and submitted for publication after this work was finished) some of us demonstrate the method of partial covariance analysis to disentangle fragment ion momenta spectra for getting insights into the Coulomb explosion of diatomic molecules exposed to intense XUV radiation fields [26]. *1.j.frasinski@imperial.ac.uk

†raimund.feifel@physics.uu.se

[1] B. W. McNeil and N. R. Thompson, Nat. Photonics 4, 814 (2010).

[2] R. Neutze, R. Wouts, D. van der Spoel, E. Weckert, and J. Hajdu, Nature (London) 406, 752 (2000).

[3] M. M. Seibert et al., Nature (London) 470, 78 (2011).

[4] J. P. Briand, L. de Billy, P. Charles, S. Essabaa, P. Briand, R. Geller, J. Desclaux, S. Bliman, and C. Ristori, Phys. Rev. Lett. 65, 159 (1990); H. Winter and F. Aumayr, J. Phys. B 32, R39 (1999), and references therein.

[5] L. Young et al., Nature (London) 466, 56 (2010).

[6] H. Schmidt-Böcking, M. Prior, R. Dörner, H. Berg, J. Pedersen, C. Cocke, M. Stockli, and A. Schlachter, Phys. Rev. A 37, 4640 (1988); B. Rudek et al., Nat. Photonics 6, 858 (2012).

[7] S. P. Hau-Riege, Phys. Rev. A 76, 042511 (2007).

[8] L. J. Frasinski, K. Codling, and P. A. Hatherly, Science 246, 1029 (1989).

[9] P. Emma et al., Nat. Photonics 4, 641 (2010).

[10] J. H. D. Eland, Adv. Chem. Phys. 141, 103 (2009).

[11] M. Kurka et al., J. Phys. B 42, 141002 (2009).

[12] J. Bozek, Eur. Phys. J. Special Topics 169, 129 (2009).

[13] J.H.D. Eland, O. Vieuxmaire, T. Kinugawa, P. Lablanquie, R. Hall, and F. Penent, Phys. Rev. Lett. 90, 053003 (2003).

[14] L. Foucar et al., Comput. Phys. Commun. 183, 2207 (2012).

[15] See Supplemental Material at http://link.aps.org/ supplemental/10.1103/PhysRevLett.111.073002 for experimental details.

[16] W.J. Krzanowski, Principles of Multivariate Analysis (Oxford University Press, New York, 1988), Chap. 14.4; K. V. Mardia, J. T. Kent, and J. M. Bibby, Multivariate Analysis (Academic Press, London, 1997), Chap. 6.5.3; T. W. Anderson, An Introduction to Multivariate Statistical Analysis (Wiley, New York, 2003), 3rd ed., Chaps. 2.5.1 and 4.3.1.

[17] K. Siegbahn, C. Nordling, G. Johansson, J. Hedman, P. F. Hedén, K. Hamrin, U. Gelius, T. Bergmark, L. O. Werme, R. Manne, and Y. Baer, ESCA Applied to Free Molecules (North Holland, Amsterdam, 1969).

[18] L. J. Frasinski, M. Stankiewicz, P. Hatherly, G. Cross, K. Codling, A. Langley, and W. Shaikh, Phys. Rev. A 46, R6789 (1992).

[19] R. J. Maurer and R. L. Watson, At. Data Nucl. Data Tables 34, 185 (1986).

[20] I. Kadar, S. Ricz, J. Végh, B. Sulik, D. Varga, and D. Berényi, Phys. Rev. A 41, 3518 (1990).

[21] N. Rohringer and R. Santra, Phys. Rev. A 76, 033416 (2007).

[22] N. Berrah et al., Proc. Natl. Acad. Sci. U.S.A. 108, 16912 (2011).

[23] Y. Hikosaka, T. Aoto, P. Lablanquie, F. Penent, E. Shigemasa, and K. Ito, Phys. Rev. Lett. 97, 053003 (2006).

[24] M. Tashiro, M. Ehara, H. Fukuzawa, K. Ueda, C. Buth, N. V. Kryzhevoi, and L. S. Cederbaum, J. Chem. Phys. 132, 184302 (2010).

[25] P. Salen et al., Phys. Rev. Lett. 108, 153003 (2012).

[26] O. Kornilov et al., J. Phys. B (to be published). 